\title{
A Class of Generalized Gough-Stewart Platforms Used for Effectively Obtaining Dynamic Isotropy - An Analytical Study
}

\author{
Behrouz Afzali-Far ${ }^{1, a}$, Per Lidström ${ }^{2}$ \\ 1Division of Machine Elements, Lund University, P.O. Box 118, Lund 22100, Sweden \\ ${ }^{2}$ Division of Mechanics, Lund University, P.O. Box 118, Lund 22100, Sweden
}

\begin{abstract}
In this paper, we propose a class of Generalized Gough-Stewart Platforms (GGSPs) used, as a novel approach, to eliminate the classical isotropic constraint of GSPs (hexapods). GGSPs are based on the standard GSP architecture with additional rotations of the three strut-pairs. Despite the architectural generalization introduced in GGSPs, they do not require much more effort in order to be fabricated. This is due to the fact that all the struts (actuators) can be chosen identical, similar to standard GSPs. We analytically show how effectively the classical isotropic constraint is removed and that still sufficient simplicity is retained. Furthermore, this paper gives an intuitive understanding of dynamic isotropy in GGSPs as well as GSPs.
\end{abstract}

\section{Introduction}

Gough-Stewart Platforms (GSPs) are perhaps the most widely used architecture of 6-degree-of-freedom parallel kinematic robots (GSPs are also known as Stewart platforms or hexapods [1]). They have several sophisticated applications including e.g. in advanced optical telescopes and 6-degree-of-freedom force sensors $[2,3]$. A GSP has a symmetric architecture where all the six base joints lie on one ring and similarly all the six platform joints lie on another ring. Fig. 1 shows a class of Generalalzied GSPs (GGSPs) and also depicts standard GSPs when $\gamma_{M}=0$.

The condition in which all the eigenfrequencies of a robot are equal is known as Complete dynamic isotropy. It is an important criterion in order to obtain dynamically optimized solutions for parallel robots. In this paper, complete dynamic isotropy is briefly mentioned as dynamic isotropy.

To obtain dynamic isotropy in GSPs, due to their architecture, a unique relation between the platform's moments of inertia has to be met. This relation is $I_{z z}^{c_{p}}=4 I_{y y}^{c_{p}}\left(I_{x x}^{c_{p}}=I_{y y}^{c_{p}}\right)$ [4]. However, in practice the inertia properties of a platform are often determined by other functional criteria related to the platform itself. Consequently, it is practically almost impossible to obtain dynamic isotropy based on the GSP architecture. Thus, the problem can be formulated as how to generalize the architecture of GSPs in order to eliminate the classical isotropic constraint $\left(I_{z z}^{c_{p}}=4 I_{y y}^{c_{p}}\right)$ and at the same time avoid introducing unnecessary complications.

Kinematics as well as equations of motion describing vibrations are required to be formulated in order to

\footnotetext{
a Corresponding author: Behrouz.afzali_far@mek.lth.se
}

analytically determine dynamic isotropy. In particular, the linearized equations of motion at the neutral configuration are of interest. These are well-known for GSPs from the literature [4-9]. However, despite the importance of dynamic isotropy as an optimal design solution, there exist a limited number of publications dealing with dynamic isotropy in GSPs. Jiang et al. [4], for the case of 3-3 GSPs ( $\delta_{b}=\delta_{p}=0$ as in [6]), parametrically formulate the conditions of dynamic isotropy. Jiang et al. [10] discuss the dynamic isotropy conditions by using a circular hyperboloid for constructing GSPs. See also [11, 12], for some special cases of dynamic isotropy.

Among the studies of generalized GSPs, Tong et al. [13] formulate a generalized architecture in which joints are arranged in two rings on the base-plane as well as two rings on the platform-plane. Jiang et al. [14] also generalize the architecture of GSPs by using a pair of circular hyperboloids. These studies discuss the dynamic isotropy conditions and categorize different families of dynamic isotropy. However, despite the value of these works, it seems that the nature of the introduced generalizations makes the problem too complicated in order to be able to draw straightforward analytical design conclusions.

In this paper, we propose a class of Generalized Gough-Stewart Platforms (GGSPs) in which each pair of struts can be rotated about a vertical axis at its corresponding mid-point $M$ on the line connecting the two joints on the platform, see Fig. 1. The proposed GGSPs can effectively eliminate the classical isotropic constraint of GSPs and still have about the same complication level as GSPs do in terms of design calculations and fabrication. Since the generalization 


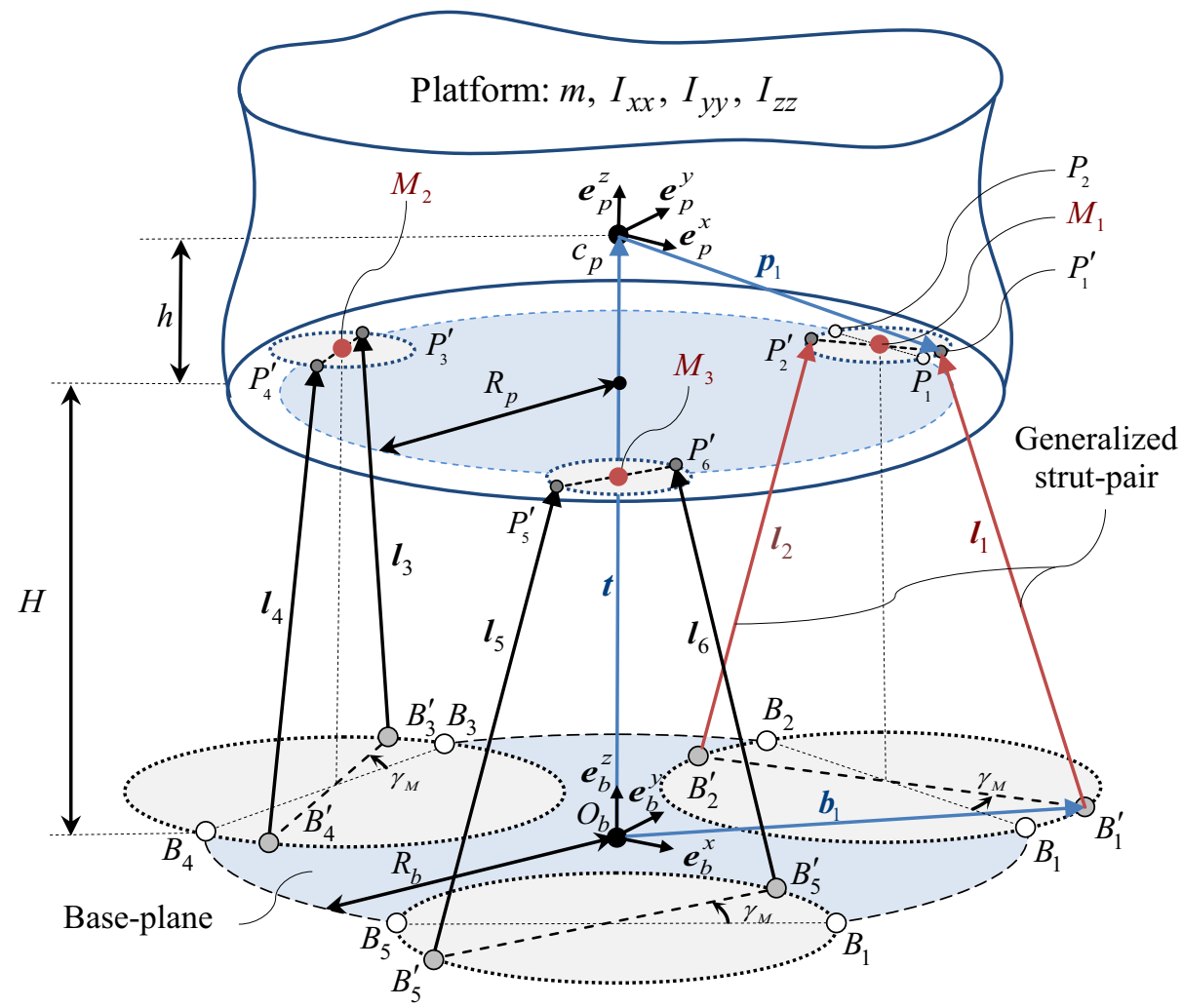

Fig. 1. Schematic of the Generalized Gough-Stewart Platform (GGSP)

introduced is based on rigid body rotations of the strutpairs, the equations can be formulated based on the variables of standard GSPs where all the initial lengths of struts are maintained identical.

\section{Kinematics}

In this paper, as indicated earlier, the aim is to firstly set up the kinematic equations of GGSPs based on the geometric variables of standard GSPs. Then, the standard GSP is generalized by introducing a new geometric variable $\gamma_{M}$ related to the rotation of the strut-pairs. Fig. 1 shows a schematic of a GGSP. See also the details regarding the GSP geometric variables in [6].

To construct the GGSP architecture, a reference strutpair is introduced, which is shown in Fig. 2. The three strut-pairs of GGSPs, shown in Fig. 1, are based on this reference strut-pair. The angle $\gamma_{M}$ represents the rotation of the strut-pairs. Considering the six GSP variables [6] as well as $\gamma_{M}$, the geometric design space $G_{s}$ for GGSPs is defined by seven geometric variables as

$$
\begin{aligned}
& G_{s}=\left\{\left(R_{b}, R_{p}, H, h, \delta_{b}, \delta_{p}, \gamma_{M}\right) \in \mathbb{R}^{7} \mid R_{b}, R_{p}, H>0,\right. \\
& \left.\delta_{b}, \delta_{p} \geq 0, \delta_{b}+\delta_{p}<\pi / 3,0 \leq \gamma_{M}<\pi / 2\right\}
\end{aligned}
$$

and in this space stiffness singularity is avoided.

As illustrated in Fig. 2, the positions of reference joints before rotation are denoted by $B, P$ and after rotation by $B^{\prime}, P^{\prime}$. Accordingly, the configuration of the reference strut-pair before rotation is defined by two vectors $\boldsymbol{r}_{B^{-} P^{-}}$and $\boldsymbol{r}_{B^{+} P^{+}}$and its configuration after rotation is given by $\boldsymbol{r}_{B^{\prime-} P^{\prime-}}$ and $\boldsymbol{r}_{B^{++} P^{\prime+}}$. The position of the reference joints before rotation are defined by

$$
\left\{\begin{array}{l}
B^{-}=\left(\begin{array}{l}
B_{x}^{-} \\
B_{y}^{-} \\
B_{z}
\end{array}\right)_{\underline{\underline{e}}_{b}}=\left(\begin{array}{c}
R_{b} \cos \left(-\pi / 3+\delta_{b}\right) \\
R_{b} \sin \left(-\pi / 3+\delta_{b}\right) \\
0
\end{array}\right)_{\underline{\boldsymbol{e}}_{b}} \\
B^{+}=\left(\begin{array}{l}
B_{x}^{+} \\
B_{y}^{+} \\
B_{z}
\end{array}\right)_{\underline{\underline{e}}_{b}}=\left(\begin{array}{c}
R_{b} \cos \left(\pi / 3-\delta_{b}\right) \\
R_{b} \sin \left(\pi / 3-\delta_{b}\right) \\
0
\end{array}\right)_{\underline{\underline{e}}_{b}}
\end{array}\right.
$$

and

$$
\left\{\begin{array}{l}
P^{-}=\left(\begin{array}{c}
P_{x}^{-} \\
P_{y}^{-} \\
P_{z}
\end{array}\right)_{\underline{e}_{b}}=\left(\begin{array}{c}
R_{p} \cos \left(-\delta_{p}\right) \\
R_{p} \sin \left(-\delta_{p}\right) \\
H
\end{array}\right)_{\underline{\underline{e}}_{b}}, \\
P^{+}=\left(\begin{array}{c}
P_{x}^{+} \\
P_{y}^{+} \\
P_{z}
\end{array}\right)_{\underline{e}_{b}}=\left(\begin{array}{c}
R_{p} \cos \left(\delta_{p}\right) \\
R_{p} \sin \left(\delta_{p}\right) \\
H
\end{array}\right)_{\underline{\boldsymbol{e}}_{b}}
\end{array}\right.
$$

where the right-handed orthonormal basis

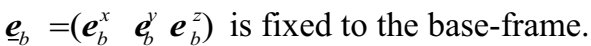

Based on the definition of the mid-point position 


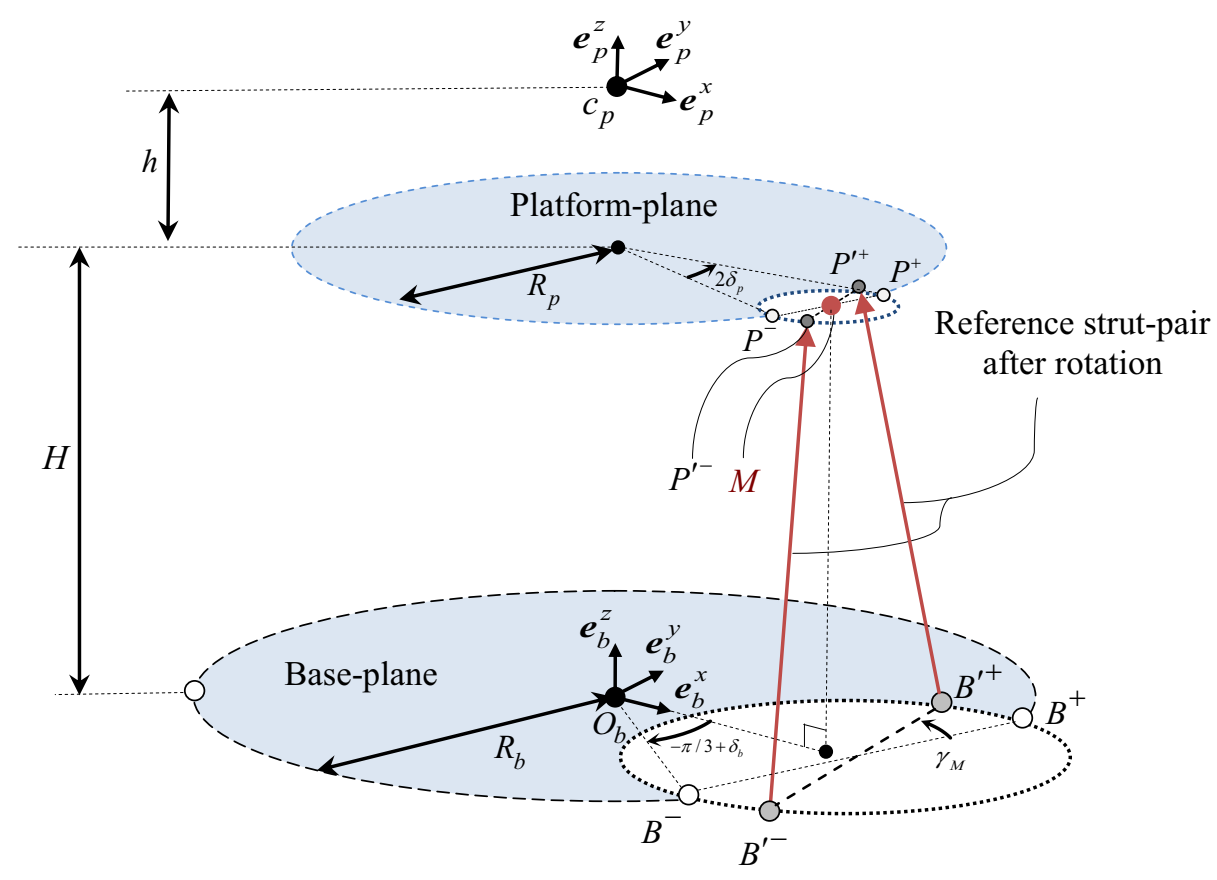

Fig. 2. Rotation of the reference strut-pair

$$
M=\frac{P^{+}+P^{-}}{2}=\left(\begin{array}{c}
R_{p} \cos \left(\delta_{p}\right) \\
0 \\
H
\end{array}\right)_{\underline{\underline{e}}_{b}},
$$

the position of the reference joints after rotation $B^{\prime}$ and $P^{\prime}$ are now calculated using

$$
\begin{gathered}
\left\{\begin{array}{l}
P^{\prime+}=\left[\boldsymbol{R}_{M}\right]_{\underline{e}_{b}}\left(P^{+}-M\right)+M \\
P^{\prime-}=\left[\boldsymbol{R}_{M}\right]_{\underline{e}_{b}}\left(P^{-}-M\right)+M
\end{array}\right. \\
\left\{\begin{array}{l}
B^{\prime+}=\left[\boldsymbol{R}_{M}\right]_{\underline{e}_{b}}\left(B^{+}-M\right)+M \\
B^{\prime-}=\left[\boldsymbol{R}_{M}\right]_{\underline{e}_{b}}\left(B^{-}-M\right)+M
\end{array}\right.
\end{gathered}
$$

where the reference strut-pair is supposed to be rotated by the rotation matrix $\left[\boldsymbol{R}_{M}\right]_{\underline{e}_{b}}$ as a rigid body about $M$. A general rotation matrix $[\boldsymbol{R}]_{\underline{e}_{b}}$ is given by

$$
\begin{array}{r}
{[\boldsymbol{R}]_{\underline{\underline{e}}_{b}}=R(\alpha, \beta, \gamma)=R_{\alpha}(\alpha) R_{\beta}(\beta) R_{\gamma}(\gamma)=} \\
c \beta c \gamma \\
c\left(\begin{array}{ccc}
c \beta & -c \beta s \gamma & s \beta \\
c \alpha s \gamma+s \alpha s \beta c \gamma & c \alpha c \gamma-s \alpha s \beta s \gamma & -s \alpha c \beta \\
s \alpha s \gamma-c \alpha s \beta c \gamma & s \alpha c \gamma+c \alpha s \beta s \gamma & c \alpha c \beta
\end{array}\right),
\end{array}
$$

where

$$
\left\{\begin{array}{l}
R_{\alpha}(\alpha)=R(\alpha, 0,0) \\
R_{\beta}(\beta)=R(0, \beta, 0) \\
R_{\gamma}(\gamma)=R(0,0, \gamma)
\end{array}\right.
$$

and the sinusoidal functions sin and $\cos$ are shortly shown by "s" and "c", respectively. Accordingly, the rotation matrix $\left[\boldsymbol{R}_{M}\right]_{\underline{\boldsymbol{e}}_{b}}$, specified in Eq. 4 , is expressed by

$$
\left[\boldsymbol{R}_{M}\right]_{\underline{\underline{e}}_{b}}=R_{\gamma}\left(\gamma_{M}\right), R_{\gamma}(\gamma)=\left(\begin{array}{ccc}
\mathrm{c} \gamma & -\mathrm{s} \gamma & 0 \\
\mathrm{~s} \gamma & \mathrm{c} \gamma & 0 \\
0 & 0 & 1
\end{array}\right) .
$$

Note that the general rotation matrix given in Eq. 5 will be used in Eq. 10 as well. This rotation matrix is based on Bryant angles due to the motivations presented in $[6,15]$. However, it could also be written based on e.g. Euler angles [16].

With a similar definition of the rotation matrix given in Eq. 7, the generalized joint positions $B_{i}^{\prime}$ and $P_{i}^{\prime}$ are calculated by

$$
\left\{\begin{array}{l}
B_{1}^{\prime}=R_{\gamma}(\pi / 3) B^{\prime-} \\
B_{2}^{\prime}=R_{\gamma}(\pi / 3) B^{\prime+} \\
B_{3}^{\prime}=R_{\gamma}(\pi) B^{\prime-} \\
B_{4}^{\prime}=R_{\gamma}(\pi) B^{\prime+} \\
B_{5}^{\prime}=R_{\gamma}(5 \pi / 3) B^{\prime-} \\
B_{6}^{\prime}=R_{\gamma}(5 \pi / 3) B^{\prime+}
\end{array},\left\{\begin{array}{l}
P_{1}^{\prime}=R_{\gamma}(\pi / 3) P^{\prime-} \\
P_{2}^{\prime}=R_{\gamma}(\pi / 3) P^{\prime^{+}} \\
P_{3}^{\prime}=R_{\gamma}(\pi) P^{\prime^{-}} \\
P_{4}^{\prime}=R_{\gamma}(\pi) P^{\prime+} \\
P_{5}^{\prime}=R_{\gamma}(5 \pi / 3) P^{\prime-} \\
P_{6}^{\prime}=R_{\gamma}(5 \pi / 3) P^{++}
\end{array},\right.\right.
$$

where $B^{\prime}$ and $P^{\prime}$ are the positions of the reference joints after rotation.

It should be emphasized that, in Eqs. 4 and 8, two types of rotations are involved in order to construct the GGSP architecture. First, there is a rotation of the reference strut-pair about $M$, which is shown in Fig. 2. Second, the reference strut-pair after rotation is again rotated about the $\boldsymbol{e}_{b}^{z}$-axis for obtaining the tri-symmetric arrangement shown in Fig. 1 (the mid-point positions of 
the three generalized strut-pairs are given by $\left.M_{1}=R_{\gamma}(\pi / 3) M, M_{2}=R_{\gamma}(\pi) M, M_{3}=R_{\gamma}(5 \pi / 3) M\right)$.

Having obtained the generalized position of the joints, the kinematic equation is written as

$$
\boldsymbol{l}_{i}=\boldsymbol{r}_{B_{i}^{\prime} P_{i}^{\prime}}=\boldsymbol{r}_{O_{b} c_{p}}+\boldsymbol{r}_{c_{p} P_{i}^{\prime}}-\boldsymbol{r}_{O_{b} B_{i}^{\prime}}
$$

and accordingly in a matrix form

$$
\left[\boldsymbol{l}_{i}\right]_{\underline{\boldsymbol{e}}_{b}}=\left[\boldsymbol{r}_{O_{b} c_{p}}\right]_{\underline{\boldsymbol{e}}_{b}}+[\boldsymbol{R}]_{\underline{\boldsymbol{e}}_{b}}\left[\boldsymbol{r}_{c_{p} P_{i}^{\prime}}\right]_{\underline{\boldsymbol{e}}_{p}}-\left[\boldsymbol{r}_{O_{b} B_{i}^{\prime}}\right]_{\underline{\boldsymbol{e}}_{b}}
$$

where $\boldsymbol{l}_{i}$ denotes the strut vectors; $[\boldsymbol{R}]_{\underline{e}_{b}}$ is the rotation matrix given in Eq. 5; the right-handed orthonormal basis $\underline{\boldsymbol{e}}_{p}=\left(\boldsymbol{e}_{p}^{x} \boldsymbol{e}_{p}^{y} \boldsymbol{e}_{p}^{z}\right)$ is fixed to the platform-frame and in the principle axes directions.

At the neutral configuration, it is assumed that the platform is not rotated. It is also assumed that $\boldsymbol{e}_{b}^{z}$-axis and $\boldsymbol{e}_{p}^{z}$-axis are collinear. Moreover, the distance between the points $c_{p}$ (platform's center of mass) and $O_{b}$ is $H+h$. Hence, the neutral configuration $q_{C, 0}$ of the platform, in the Cartesian space $q_{C}$, is defined by

$$
q_{C, 0}=\left(\begin{array}{llllll}
0 & 0 & H+h & 0 & 0 & 0
\end{array}\right)^{T}
$$

where in general $q_{C}=\left(\begin{array}{llllll}t_{x} & t_{y} & t_{z} & \alpha & \beta & \gamma\end{array}\right)^{T}$ and

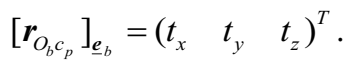

\section{Stiffness}

The stiffness matrix can be calculated directly using the Jacobian matrix [6]. The Jacobian matrix $J$ is defined as the transformation between the Cartesian velocities $\dot{q}_{C}$ of the platform and the length rates $\dot{q}_{J}$ of the struts $\left(q_{J}=\left(\begin{array}{llllll}l_{1} & l_{2} & l_{3} & l_{4} & l_{5} & l_{6}\end{array}\right)^{T}, l_{i}=\left|\boldsymbol{l}_{i}\right|\right)$,

$$
\begin{gathered}
J=\left.\left[\frac{\dot{q}_{J}}{\dot{q}_{C}}\right]\right|_{q_{C, 0}}=\left.\left[\frac{\partial q_{J}}{\partial q_{C}}\right]\right|_{q_{C, 0}} \in \mathbb{R}^{6 \times 6}, \\
j_{i k}=\left.\left[\frac{1}{2 l_{i, 0}} \frac{\partial l_{i}^{2}}{\partial q_{C, k}}\right]\right|_{q_{C, 0}}=\left.\frac{1}{2 l_{0}}\left\{\frac{\partial\left(\left[\boldsymbol{l}_{i}\right]_{\underline{e}_{b}}^{T}\left[\boldsymbol{l}_{i}\right]_{\underline{e}_{b}}\right)}{\partial q_{C, k}}\right\}\right|_{q_{C, 0}}
\end{gathered}
$$

where the neutral configuration $q_{C, 0}$ is that given in Eq. $11 ; q_{C, k}$ denotes each coordinate of the Cartesian space $q_{C}=\left(\begin{array}{lllllll}t_{x} & t_{y} & t_{z} & \alpha & \beta & \gamma\end{array}\right)^{T} \quad$ for $\quad k=1, \ldots, 6 \quad ; \quad j_{i k}$ denotes the components of the Jacobian matrix and the column matrix $\left[\boldsymbol{l}_{i}\right]_{\underline{\underline{e}}_{b}}$ is that given in Eq. 10. Note that, according to the architecture of GGSPs, the neutral lengths of all struts are equal $\left(l_{i, 0}=l_{0}\right)$.
Using Eq. 12, the Cartesian-space stiffness matrix $K_{C}$ can be calculated parametrically by

$$
K_{C}=J^{T} K_{J} J \in \mathbb{R}^{6 \times 6}, K_{J}=\operatorname{diag}(k \quad \ldots \quad k) \in \mathbb{R}^{6 \times 6},
$$

where $K_{J}$ is the joint-space stiffness matrix. Accordingly, the Cartesian-space stiffness matrix is calculated and structured as

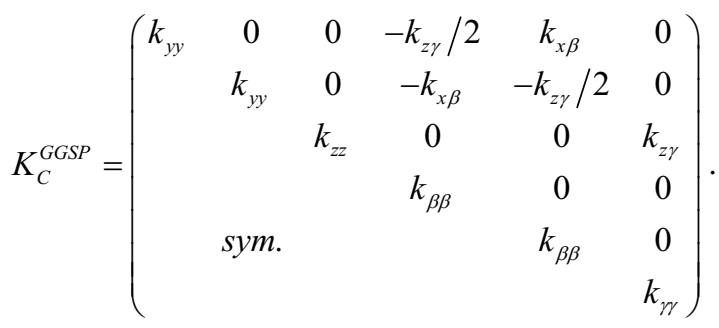

In the following section, we study the diagonalization of this matrix and the conditions for obtaining dynamic isotropy in GGSPs.

\section{Dynamic isotropy}

Dynamic isotropy is obtained when the matrix $M^{-1} K$ becomes a scaled identity according to

$$
M^{-1} K=\sigma I \Leftrightarrow K=\sigma M, \sigma \in \mathbb{R}^{+},
$$

from which it follows that all the eigenvalues of the matrix $M^{-1} K$ are equal. In the joint space, this condition is simpler due to the fact that the stiffness matrix $K_{J}$, given in Eq. 13, is already a scaled identity matrix. Hence, in the joint space, only the inertia matrix is required to be a scaled identity matrix in order to obtain dynamic isotropy, see [17].

The base-frame basis $\underline{\boldsymbol{e}}_{b}$ is chosen to be initially in line with the principle axes of the platform, as shown in Fig. 1. Thus, the inertia matrix in the Cartesian space is diagonal and is given by [6]

$$
M_{C}=\operatorname{diag}\left(\begin{array}{cccccc}
m & m & m & I_{x x}^{c_{p}} & I_{y y}^{c_{p}} & I_{z z}^{c_{p}}
\end{array}\right) \in \mathbb{R}^{6 \times 6} .
$$

According to Eq. 15, the stiffness matrix is required to be proportional to the inertia matrix. Hence, since the Cartesian-space inertia matrix $M_{C}$ is diagonal, the first step in order to obtain dynamic isotropy is to diagonalize the Cartesian-space stiffness matrix $\left(k_{z \gamma}=0, k_{x \beta}=0\right)$. Subsequently, with the consideration of Eq. 15, the dynamic isotropy conditions are given by

$$
\frac{k_{y y}}{m}=\frac{k_{z z}}{m}=\frac{k_{\beta \beta}}{I_{x x}^{c_{p}}}=\frac{k_{\beta \beta}}{I_{y y}^{c_{p}}}=\frac{k_{\gamma \gamma}}{I_{z z}^{c_{p}}}, k_{z \gamma}=0, k_{x \beta}=0,
$$

from which it directly follows that $I_{x x}^{c_{p}}=I_{y y}^{c_{p}}$. Only three of the conditions given in Eq. 17 impose purely geometric constraints $\left(k_{y y}=k_{z z}, k_{z \gamma}=0\right.$ and $\left.k_{x \beta}=0\right)$. 
Purely geometric constraints are those which are independent of the inertia conditions.

To obtain dynamic isotropy in GGSPs, there exist totally six constraint equations which are given in Eq. 17. However, this is not the case for GSPs. If $\gamma_{M}=0$, where the GGSP is reduced to a GSP, the structure of the stiffness matrix is simpler which is shown in [6]. Due to this simpler structure, the condition $k_{z \gamma}=0$ is already satisfied. This means that one geometric constraint equation is eliminated. Therefore, for GSPs there are five constraint equations, of which only two of them $\left(k_{y y}=k_{z z}, k_{x \beta}=0\right)$ are purely geometric.

Having solved the system of equations given in Eq. 17 , the end result regarding the inertia condition is obtained. Accordingly, the most important property of GGSPs is that the inertia condition $k_{\gamma \gamma} / I_{z z}^{c_{p}}=k_{\beta \beta} / I_{y y}^{c_{p}}=k_{\beta \beta} / I_{x x}^{c_{p}}$ becomes

$$
I_{z z}^{c_{p}}=\left[4 \cos ^{2}\left(\gamma_{M}\right)\right] I_{y y}^{c_{p}}=\left[4 \cos ^{2}\left(\gamma_{M}\right)\right] I_{x x}^{c_{p}}
$$

which is a simple equation that replaces the strict classical constraint $\left(I_{z z}^{c_{p}}=4 I_{y y}^{c_{p}}=4 I_{x x}^{c_{p}}\right)$ by a constraint involving an arbitrary choice of $\gamma_{M}$. Interestingly, the relation obtained in Eq. (18) is in line with the results obtained from a study on 2-2-2 kinematic arrangements [18].

As a numeric example, another inertia condition of the platform such as $I_{z z}^{c_{p}}=I_{y y}^{c_{p}}=I_{x x}^{c_{p}}$ (instead of $\left.I_{z z}^{c_{p}}=4 I_{y y}^{c_{p}}=4 I_{x x}^{c_{p}}\right)$ can be easily accomplished by taking $\gamma_{M}=\pi / 3$ in GGSPs. This condition is fundamentally impossible to obtain in standard GSPs. Therefore, the proposed GGSP and its analytical study presented in this paper can be directly employed in order to obtain dynamic isotropy for a wide range of inertia conditions where $0<I_{z z}^{c_{p}} \leq 4 I_{y y}^{c_{p}}\left(I_{x x}^{c_{p}}=I_{y y}^{c_{p}}\right)$.

\section{Conclusion}

In this paper, a novel generalized GSP is proposed in order to eliminate the classical isotropic constraint of GSPs $\left(I_{z z}^{c_{p}}=4 I_{y y}^{c_{p}}\right)$. It is shown how effectively this isotropic constraint can be eliminated only by finding a corresponding value for the rotation angle $\gamma_{M}$ in GGSPs. Moreover, the fabrication of GGSPs is quite similar to GSPs, since the struts (actuators) can still be chosen identical in spite of the generalization. It is also shown that for GGSPs, which have seven geometric variables along with the inertia variables, there exist six constraint equations for obtaining dynamic isotropy. Three of the six constraint equations are purely geometric and independent of the inertia conditions. It is concluded that the introduced GGSP is perhaps the simplest architecture of generalized GSPs in which the classical constraint is removed. In addition, this paper gives an intuitive understanding of the problem of dynamic isotropy in GGSPs as well as GSPs.

\section{References}

1. B. Dasgupta, T.S. Mruthyunjaya: Mechanism and Machine Theory, 35 (2000), p. 15.

2. B. Afzali-Far, P. Lidström, K. Nilsson, A. Ardeberg: Proceedings of the 25th Nordic Seminar on Computational Mechanics, (2012), p. 257.

3. J.T. Yao, Y.L. Hou, H. Wang, T.L. Zhou, Y.S. Zhao: Mechanism and Machine Theory, 46 (2011), p. 142.

4. H.Z. Jiang, J.F. He, Z.Z. Tong: Mechanism and Machine Theory, 45 (2010), p. 722.

5. B. Afzali-Far, P. Lidström: Progress in Systems Engineering, Springer International Publishing, (2015), p. 323.

6. B. Afzali-Far, P. Lidström, K. Nilsson: Mechanism and Machine Theory, 80 (2014), p. 52.

7. S. Pedrammehr, M. Mahboubkhah, N. Khani: International Journal of Advanced Manufacturing Technology, 65 (2013), p. 991.

8. M. Mahboubkhah, M.J. Nategh, S.E. Khadem: International Journal of Advanced Manufacturing Technology, 38 (2008), p. 1236.

9. J. Chen, F. Lan: Simulation Modelling Practice and Theory, 16 (2008), p. 419.

10. H.Z. Jiang, Z.Z. Tong, J.F. He: Mechanism and Machine Theory, 46 (2011), p. 358.

11. J.E. McInroy, J.F. O'Brien, A.A. Allais: 2013 Ieee International Conference on Robotics and Automation (Icra), (2013), p. 1458.

12. J.F. He, H.Z. Jiang, Z.Z. Tong, B.P. Li, J.W. Han: Journal of Vibration and Control, 18 (2012), p. 1156.

13. Z.Z. Tong, J.F. He, H.Z. Jiang, G.R. Duan: Robotica, 30 (2012), p. 305.

14. H.Z. Jiang, J.F. He, Z.Z. Tong, W. Wang: Mechanism and Machine Theory, 46 (2011), p. 1301.

15. B. Afzali-Far, A. Andersson, K. Nilsson, P. Lidstrom: Journal of Sound and Vibration, 352 (2015), p. 142.

16. B. Afzali-Far, P. Lidström: Mathematics and Mechanics of Solids, 1081286514546180 (2014), p. 1.

17. O. Ma, J. Angeles: Ieee International Conference on Robotics and Automation, (1993), p. 470.

18. B. Afzali-Far, A. Andersson, K. Nilsson, P. Lidstrom: submitted to Precision Engineering - Journal of the International Societies for Precision Engineering and Nanotechnology (2015). 